\title{
Differing roles of autophagy in HIV-associated neurocognitive impairment and encephalitis with implications for morphine co-exposure
}

OPEN ACCESS

Edited by:

Pankaj Seth,

National Brain Research Centre, India

Reviewed by:

Fatah Kashanchi,

George Mason University, USA

Sulie L. Chang,

Seton Hall University, USA

Sanjay Balkrishna Maggirwar,

University of Rochester Medical

Center, USA

*Correspondence:

Nazira El-Hage,

Department of Immunology, Herbert Wertheim College of Medicine, Florida

International University,

11200 Southwest 8th Street, Miami,

FL 33199, USA

nelhage@fiu.edu

Specialty section:

This article was submitted to

Virology,

a section of the journal

Frontiers in Microbiology

Received: 15 January 2015

Accepted: 15 June 2015

Published: 06 July 2015

Citation:

Dever SM, Rodriguez M, Lapierre J, Costin BN and El-Hage N (2015)

Differing roles of autophagy in HIV-associated neurocognitive impairment and encephalitis with implications for morphine co-exposure.

Front. Microbiol. 6:653. doi: 10.3389/fmicb.2015.00653
Seth M. Dever ${ }^{1,2}$, Myosotys Rodriguez ${ }^{2}$, Jessica Lapierre ${ }^{2}$, Blair N. Costin ${ }^{1}$ and Nazira El-Hage ${ }^{1,2 *}$

${ }^{1}$ Department of Pharmacology and Toxicology, School of Medicine, Virginia Commonwealth University, Richmond, VA, USA, ${ }^{2}$ Department of Immunology, Herbert Wertheim College of Medicine, Florida International University, Miami, FL, USA

We investigated the role of autophagy in HIV-infected subjects with neurocognitive impairment $(\mathrm{NCl}) \pm \mathrm{HIV}$ encephalitis (HIVE), many of which had a history of polysubstance abuse/dependence, using post-mortem brain tissues to determine whether differences in autophagy related factors may be more associated with $\mathrm{NCl}$ or $\mathrm{NCl}$-encephalitis. Using qRT-PCR, we detected significant differences in gene expression levels with SQSTM1, LAMP1 higher in HIV-infected subjects without $\mathrm{NCl}$ while ATG5, SQSTM1 were then lower in HIV infection/NCI and ATG7, SQSTM1 being higher in NCl-HIVE. Immunohistochemical labeling of these autophagy associated proteins (also including Beclin 1 and LC3B) in Iba1-positive microglial cells showed generally higher immunoreactivity in the $\mathrm{NCl}$ and $\mathrm{NCl}-\mathrm{HIVE}$ groups with more focal vs. diffuse patterns of expression in the NCI-HIVE group. Furthermore, analysis of microarray data from these same subjects found significantly higher levels of LAMP1 in $\mathrm{NCl}-\mathrm{HIVE}$ compared to uninfected subjects in the basal ganglia. Finally, we tested the effect of supernatant from HIV-1-infected microglia and HIV-1 Tat protein in combination with morphine on neurons in vitro and found opposing events with both significant inhibition of autophagic flux and reduced dendrite length for morphine and supernatant treatment while Tat and morphine exposure resulted in lower autophagic activity at an earlier time point and higher levels in the later. These results suggest autophagy genes and their corresponding proteins may be differentially regulated at the transcriptional, translational, and post-translational levels in the brain during various stages of the HIV disease and that infected individuals exposed to morphine can experience mixed signaling of autophagic activity which could lead to more severe $\mathrm{NCl}$ than those without opioid use.

Keywords: autophagy, HIV-associated neurocognitive disorders, HIV encephalitis, microarray, microglia, neuron, morphine 


\section{Introduction}

The central nervous system (CNS) is preferentially vulnerable to human immunodeficiency virus (HIV) infection as chronic exposure to HIV in the brain can lead to neurocognitve impairment (NCI) from HIV-associated neurocognitive disorders (HANDs; Nabha et al., 2013). HAND can range from asymptomatic neurocognitive impairment (ANI) intermediately to HIV-associated mild neurocognitive disorder [MND; formerly referred to as minor cognitive and motor disorder (MCMD)] to the most severe condition of HIV-associated dementia (HAD; Antinori et al., 2007; Woods et al., 2009), manifested pathologically as HIV encephalitis (HIVE; Zink et al., 1999), through interactive cellular events (Kaul et al., 2005). As neurons have not generally been found to be infected by HIV (Kramer-Hammerle et al., 2005; Verma et al., 2010), most of the HIV-associated neurotoxicity can occur from bystander effects through the actions of infected/activated glia (Kaul et al., 2001). Microglia are the primary target of HIV in the brain (Kramer-Hammerle et al., 2005), and this cell type can release various toxic and inflammatory factors during the course of infection resulting in neuronal injury leading to HAD (Anderson et al., 2002; Garden, 2002; Gonzalez-Scarano and Martin-Garcia, 2005).

Macroautophagy (hereafter referred to as autophagy) is a critical mechanism to ensure proper homeostasis and functioning of the cell through removal of unused and damaged cellular organelles and components. In the process of autophagy, autophagosomes carrying these contents fuse with lysosomes creating post-fusion autolysosomes during autophagic flux in which the contained cellular material is degraded and recycled (Klionsky and Emr, 2000). Although disruptions to autophagy in neuronal cells have been implicated in neurodegenerative diseases (Komatsu et al., 2007a; Cherra and Chu, 2008), less is known about the role of autophagy in microglia and how modulation of autophagic processes in microglial cells may intercellularly lead to alterations of this and other pathways in bystander cell types such as neurons, particularly in the context of HIV infection. While autophagy may be thought of as being a generally cytoprotective process in response to various factors (Moreau et al., 2010), it appears to play a complex role in viral replication with cells permissive to productive HIV infection being both induced (Wang et al., 2012), and inhibited (Zhou and Spector, 2008), which may be selectively occurring to promote virus production at different stages of the viral life cycle (Espert et al., 2009). However, initial compensatory higher levels of autophagic activity in bystander cells such as neurons to remove viral-induced toxins followed by cellular differences resulting in reduced or defective autophagy may lead to neurodegeneration and neurocognitive deficits if an accumulation of protein aggregates results from the process not being driven to completion (Gelman and Schuenke, 2004; Yu et al., 2005).

The autophagic machinery consists of a variety of autophagyrelated $(A T G)$ genes and their encoded protein products (ATG; Klionsky, 2012). Beclin 1 (ATG6) is involved in the initiation of autophagosome formation (Liang et al., 1999), while the yeast Atg8 mammalian homolog LC3, of which LC3B is the most commonly examined isoform (Mizushima et al., 2010), associates with the autophagosomal membrane to promote its elongation and maturation following phosphatidylethanolamine conjugation in the conversion of LC3-I to the lipidated form LC3II through ubiquitin-like reactions (Kabeya et al., 2000, 2004). ATG7 acts as an E1-like enzyme involved in formation of the ATG12-ATG5 conjugate (Tanida et al., 2001, 2012), which then acts as an E3-like enzyme in the LC3 lipidation reaction (Hanada et al., 2007). In addition to components directly involved in the autophagic process, markers of autophagic activity have also been identified. Sequestosome 1 (p62/SQSTM1) binds to Atg8/LC3 and disruptions in autophagy have been found to be associated with higher p62/SQSTM1 expression levels (Bjorkoy et al., 2005; Wang et al., 2006; Komatsu et al., 2007b; Pankiv et al., 2007), while higher lysosomal-associated membrane protein 1 (LAMP1) can indicate the presence of more lysosomes and autophagosomes during rises in autophagic activity (Klionsky et al., 2008).

While cellular differences resulting from prolonged HIV infection can result in NCI, drugs of abuse such as opiates can exacerbate and accelerate the onset of these deficits (Hauser et al., 2012). HIV/AIDS and opiate drug abuse are interlinked epidemics among injection drug users (Leshner, 1998), and morphine, the main bioactive product of heroin in the brain (Wright, 1940; Jaffe and Martin, 1985), can enhance HIV-1 replication (Steele et al., 2003), as well as neuronal toxicity in the presence of HIV-1 Tat and gp120 proteins (Zou et al., 2011; Podhaizer et al., 2012). However, whether alterations to autophagy may be a contributing factor in these interactive effects is largely unknown.

In the present study, we examined the role of autophagy in HIV-associated NCI using post-mortem brain tissues from HIV-infected subjects with NCI \pm HIVE as well as unimpaired infected subjects, many of which had a history of polysubstance abuse/dependence, to determine whether the differences that we found were more associated with impairment or encephalitis. We also performed in vitro studies to examine the effect of HIV-morphine interactions on autophagy in primary human neurons. The findings suggest mixed autophagic signals in the HIV-infected brain may be a mechanism resulting in cellular dysfunction leading to NCI that is more associated with HIVE than NCI alone. Additionally, supernatant from HIV-1-infected primary human microglia and HIV-1 Tat protein in combination with morphine can modify the autophagic activity of neurons in opposing ways which could accelerate the onset of these deficits.

\section{Materials and Methods}

\section{Human Brain Tissue}

Human brain tissue was obtained from a subset of samples used in the National NeuroAIDS Tissue Consortium (NNTC) Gene Array Project (Morgello et al., 2001; Gelman et al., 2012). 
Briefly, the array project consists of four groups of subjects [HIVnegative (Group A), $n=6$; HIV-positive (Group B), $n=6$; HIV-positive with neurocognitive impairment (NCI; Group C), $n=7$; and HIV-positive with combined NCI and HIVE (Group D), $n=5$ ] with post-mortem tissue samples taken from three brain regions across the groups [the frontal lobe white matter, $n=9$; frontal cortex, $n=10$; and basal ganglia, $n=1$ (or combined frontal lobe white matter/frontal cortex tissue, $n=8$ ), where $n=$ the total number of samples obtained for all groups combined]. Details on the specific brain regions and numbers of individual samples analyzed for each subject group in this study as well as age, neurocognitive diagnosis, brain pathology, and substance use histories for each subject are listed in Table $\mathbf{1}$ and have been described previously (Dever et al., 2012, 2014).

\section{Quantitative Real Time-Polymerase Chain Reaction}

Total RNA was isolated using the miRNeasy Mini Kit (Qiagen; Valencia, CA, USA) and used to generate cDNA templates by reverse transcription of $1 \mu \mathrm{g}$ RNA using the High Capacity cDNA Reverse Transcription Kit (Applied Biosystems; Carlsbad, CA, USA) according to the manufacturer's instructions. PCR reactions were performed in a total volume of $20 \mu \mathrm{L}$ containing SensiMix SYBR qPCR reagents (Bioline; Tauton, MA, USA) using a Corbett Rotor-Gene 6000 real-time PCR system (Qiagen). PCR conditions consisted of an initial hold step at $95^{\circ} \mathrm{C}$ for $10 \mathrm{~min}$ followed by 40 amplification cycles of $95^{\circ} \mathrm{C}$ for $10 \mathrm{~s}, 58^{\circ} \mathrm{C}$ for $30 \mathrm{~s}$, and $72^{\circ} \mathrm{C}$ for $30 \mathrm{~s}$. Sequences of the primer sets used are listed in Table 2 . The specificity of the amplified products was verified by melting curve analysis and agarose gel electrophoresis. qRT-PCR data were calculated as relative expression levels by normalization to GAPDH mRNA using the $2^{-\Delta \Delta \mathrm{Ct}}$ method (Livak and Schmittgen, 2001).

\section{Immunohistochemistry}

Frozen brain tissue from the NNTC Gene Array Project subjects was sectioned to 5 micron thickness, fixed in $4 \%$ paraformaldehyde, permeabilized with $0.5 \%$ Triton X-100, blocked in $10 \%$ milk $/ 0.1 \%$ goat serum, and immunolabeled. Primary antibodies used were anti-p62/SQSTM1 (catalog number NBP1-48320) at a 1:100 dilution and anti-Beclin 1 (catalog number NB500-249), anti-LC3B (catalog number NB600-1384), anti-ATG5 (catalog number NB110-53818), and anti-LAMP1 (catalog number NB120-19294) at a 1:400 dilution from Novus Biologicals (Littleton, CO, USA); antiAPG7/ATG7 (Santa Cruz Biotechnology; Santa Cruz, CA, USA; catalog number sc-33211) at a 1:100 dilution; and antiIbal (Abcam; Cambridge, MA, USA; catalog number ab5076) at a 1:100 dilution. Immunoreactivity was visualized with secondary antibodies from Molecular Probes (Carlsbad, CA, USA) conjugated to Alexa Fluor 488 dye (catalog number A11055) for Iba1 and Alexa Fluor 594 dye (catalog number A11037) for autophagy proteins and related markers, both used at a 1:200 dilution. DAPI staining was used to label cell nuclei.

\section{Microarray Data Analysis}

National NeuroAIDS Tissue Consortium Gene Array Project CEL files for individual arrays were retrieved from the NCBI Gene Expression Omnibus ${ }^{1}$, GEO accession number GSE35864 (Gelman et al., 2012), and re-analyzed as described previously (Dever et al., 2014). Briefly, robust multi-array average (RMA) analysis for probe intensity data normalization (Irizarry et al., 2003), and multi-class linear models for microarray data (Limma) analysis to access differential expression between the subject groups (Smyth, 2004), were performed for each brain region. Limma output $p$-values were adjusted by Benjamini and Hochberg's (1995) false discovery rate correction for multiple testing, setting the significance level to 0.05 . Heat maps were constructed from probeset RMA values using MultiExperiment Viewer $4.9^{2}$ (Saeed et al., 2003, 2006).

\section{Preparation of Supernatant from HIV-1-Infected Microglia}

Primary human microglia were purchased from ScienCell Research Laboratories (Carlsbad, CA, USA; catalog number 1900-f1) and cultured according to the manufacturer's instructions. Cells were infected with the macrophage-tropic HIV-1 SF 162 strain [obtained through the NIH AIDS Reagent Program, Division of AIDS, NIAID, NIH: HIV-1 SF 162 from Dr. Jay Levy (Cheng-Mayer and Levy, 1988)] at a concentration of $50 \mathrm{pg} \mathrm{HIV}-1 \mathrm{p} 24 / 10^{6}$ cells as performed previously (El-Hage et al., 2013, 2014, 2015). Seven days post-infection, the cell culture supernatant was collected and passed through a $0.20 \mu \mathrm{m}$ filter. Productive infection was confirmed and HIV-1 p24 protein levels were measured in supernatants using the HIV-1 p24 Antigen Capture Assay (Advanced Bioscience Laboratories; Rockville, MD, USA; catalog number 5421).

\section{Neuronal Cell Treatment and Immunocytochemistry}

Primary human neurons were purchased from ScienCell Research Laboratories (catalog number 1520) and cultured according to the manufacturer's instructions. Cells were treated with supernatants from uninfected or HIV-1-infected $(30 \mathrm{pg} / \mathrm{mL}$ HIV-1 p24) microglia and $500 \mathrm{nM}$ morphine sulfate (SigmaAldrich; St. Louis, MO, USA; catalog number M8777) alone or in combination for $24 \mathrm{~h}$ and fixed in $4 \%$ paraformaldehyde, permeabilized with $0.5 \%$ Triton X-100, blocked in $10 \%$ milk/0.1\% goat serum, and immunolabeled. Primary antibodies were antip62/SQSTM1 (Novus Biologicals; catalog number NBP1-48320) and anti-MAP2 (Abcam; catalog number ab5392), both used at a 1:100 dilution. Immunoreactivity was visualized with secondary antibodies from Molecular Probes conjugated to Alexa Fluor 594 dye (catalog number A11037) for p62/SQSTM1 and Alexa Fluor 488 dye (catalog number A11039) for MAP2, both used at a 1:500 dilution. DAPI staining was used to label cell nuclei.

\footnotetext{
${ }^{1}$ http://www.ncbi.nlm.nih.gov/geo/

${ }^{2}$ http://www.tm4.org/mev.html
} 
TABLE 1 | Sample origins, neurocognitive diagnoses, and substance use histories of subjects.

\begin{tabular}{|c|c|c|c|c|c|}
\hline ID & Age & Sample origin & $\begin{array}{l}\text { Neurocognitive } \\
\text { diagnosis }^{b}\end{array}$ & Brain pathologyc & PRISM/CIDI substance use history ${ }^{d}$ \\
\hline A1 & 44 & Frontal lobe white matter & Normal & No path & Not assessed \\
\hline A2 & 53 & Frontal lobe white matter & Normal & No path & Not assessed \\
\hline A3 & 63 & Frontal lobe white matter & Normal & No path & Not assessed \\
\hline A4 & 58 & $\begin{array}{l}\text { Combined frontal lobe white } \\
\text { matter/frontal cortex }\end{array}$ & Normal & No path & Not assessed \\
\hline A5 & 34 & $\begin{array}{l}\text { Combined frontal lobe white } \\
\text { matter/frontal cortex }\end{array}$ & Normal & No path & Not assessed \\
\hline A6 & 48 & Frontal cortex & Normal & No path & Not assessed \\
\hline B1 & 49 & Frontal lobe white matter & Normal & No path & Cannabis dependence, opiate dependence \\
\hline B2 & 47 & $\begin{array}{l}\text { Combined frontal lobe white } \\
\text { matter/frontal cortex }\end{array}$ & Normal & No path & $\begin{array}{l}\text { Alcohol abuse/dependence, cannabis } \\
\text { abuse/dependence, cocaine abuse, hallucinogen } \\
\text { abuse, opiate abuse, sedative abuse, stimulant } \\
\text { abuse }\end{array}$ \\
\hline B3 & 44 & $\begin{array}{l}\text { Combined frontal lobe white } \\
\text { matter/frontal cortex }\end{array}$ & Normal & No path & No history reported \\
\hline B4 & 59 & Frontal cortex & Subsyndromic & No path & Alcohol abuse \\
\hline B5 & 60 & Frontal cortex & Normal & No path & $\begin{array}{l}\text { Cannabis abuse, cocaine abuse/dependence, } \\
\text { hallucinogen abuse, stimulant abuse/dependence, } \\
\text { other drug abuse/dependence }\end{array}$ \\
\hline B6 & 39 & $\begin{array}{l}\text { Frontal lobe white matter } \\
\text { Frontal cortex }\end{array}$ & Normal & Minimal non-diagnostic abnormalities & No history reported \\
\hline C1 & 33 & Frontal lobe white matter & "Possible HAD" & Minimal non-diagnostic abnormalities & Cannabis dependence, cocaine dependence \\
\hline $\mathrm{C} 2$ & 35 & $\begin{array}{l}\text { Combined frontal lobe white } \\
\text { matter/frontal cortex }\end{array}$ & MCMD & Alzheimer's type 2 gliosis, focal infarct & No history reported \\
\hline C3 & 57 & $\begin{array}{l}\text { Combined frontal lobe white } \\
\text { matter/frontal cortex }\end{array}$ & "Possible HAD" & Atherosclerosis of brain & $\begin{array}{l}\text { Alcohol abuse/dependence, stimulant } \\
\text { abuse/dependence }\end{array}$ \\
\hline C4 & 36 & Frontal cortex & MCMD & Lymphoma & Not assessed \\
\hline C5 & 52 & Frontal cortex & MCMD & No path & Alcohol abuse/dependence \\
\hline C6 & 41 & $\begin{array}{l}\text { Frontal lobe white matter } \\
\text { Frontal cortex }\end{array}$ & "Probable HAD" & Minimal non-diagnostic abnormalities & Not assessed \\
\hline C7 & 52 & $\begin{array}{l}\text { Frontal lobe white matter } \\
\text { Frontal cortex } \\
\text { Basal ganglia }\end{array}$ & HAD & Other non-infectious path & $\begin{array}{l}\text { Alcohol abuse/dependence, hallucinogen abuse, } \\
\text { sedative abuse, stimulant abuse/dependence, } \\
\text { other drug abuse }\end{array}$ \\
\hline D1 & 48 & Frontal lobe white matter & "Probable MCMD" & HIVE & Cocaine dependence, sedative dependence \\
\hline D2 & 32 & $\begin{array}{l}\text { Combined frontal lobe white } \\
\text { matter/frontal cortex }\end{array}$ & $\mathrm{HAD}$ & HIVE, microglial nodule encephalitis & $\begin{array}{l}\text { Alcohol abuse/dependence, opiate } \\
\text { abuse/dependence, sedative abuse/dependence }\end{array}$ \\
\hline D3 & 39 & $\begin{array}{l}\text { Combined frontal lobe white } \\
\text { matter/frontal cortex }\end{array}$ & $\mathrm{HAD}$ & No path & No history reported \\
\hline D4 & 55 & Frontal cortex & $\mathrm{HAD}$ & HIVE & Not assessed \\
\hline D5 & 41 & Frontal cortex & "Probable HAD" & $\begin{array}{l}\text { CMV encephalitis, HIVE, Iymphoma, } \\
\text { microglial nodule encephalitis, other } \\
\text { infections, PML }\end{array}$ & Not assessed \\
\hline
\end{tabular}

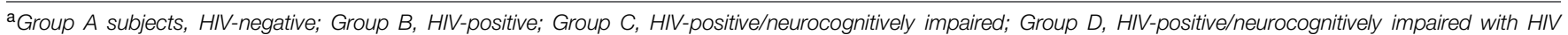
encephalitis (HIVE).

b Subsyndromic, mildy abnormal test performance without symptoms of impairment; HAD, HIV-associated dementia; MCMD, minor cognitive and motor disorder.

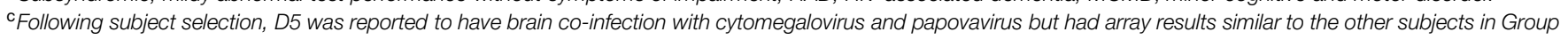
D on a principal components analysis and therefore remained included in this and the original study (Gelman et al., 2012).

dPRISM, Psychiatric Research Interview for Substance and Mental Disorders; CIDI, Composite International Diagnostic Interview.

\section{Neuronal Cell Transfection and Treatment}

Neurons were transfected with the mRFP-GFP-LC3 tandem fluorescently tagged LC3 plasmid ptfLC3 [Addgene; Cambridge, MA, USA; catalog number 21074 (Kimura et al., 2007)] using Lipofectamine 2000 reagent (Invitrogen; Carlsbad, CA, USA). Fourty-eight hours post-transfection, cells were treated with 100 nM HIV-1 IIIB recombinant Tat ${ }_{1-86}$ protein (ImmunoDX; Woburn, MA, USA; catalog number 1002-2) and $500 \mathrm{nM}$ morphine alone or in combination for $8 \mathrm{~h}$. Cells were fixed in $3.7 \%$ paraformaldehyde and stained with DAPI to label cell nuclei.

\section{Confocal Microscopy and Image Analysis}

A Zeiss LSM 700 confocal laser scanning microscope (Carl Zeiss; Thornwood, NY, USA) was used to image tissue sections with a $63 \mathrm{x}$ oil immersion objective and transfected 
TABLE 2 | Primer sets used for qRT-PCR.

\begin{tabular}{lll}
\hline Gene & Forward primer & Reverse primer \\
\hline BECN1 & 5'- GCTGAGAGACTGGATCAGGA -3' & 5'- ATTGTGCCAAACTGTCCACT -3' \\
MAP1LC3B & 5'- GAATCTCCCACACCAAGTG -3' & 5'- AAATAGTGAACCCCATGCAA -3' \\
ATG7 & 5'- TGTGTTGGAGATTGGTCCT -3' & 5'- GAGATCTTGGCATTGTCCAC -3' \\
ATG5 & 5'- ATGCAGGGAACACTAAGCTG -3' & 5'- TCTAGGGCATTGTAGGCTTG -3' \\
SQSTM1 & 5'- GAGTTCCAGCACAGAGGAGA -3' & 5'- AAGACAGATGGGTCCAGTCA -3' \\
LAMP1 & 5'- AGTGTCTGCTGGACGAGAAC -3' & 5'- GACCCTAAGCCCAGAGAAAG -3' \\
GAPDH & 5'- CATGGCACCGTCAAGGCTGAGAA -3' & 5'- CAGTGGACTCCACGACGTACTCA -3' \\
\hline
\end{tabular}

neurons at 20x magnification. Neurons treated with microglial supernatants were imaged using a Zeiss LSM 710 confocal laser scanning microscope (Carl Zeiss) at 20x magnification. Images were collected using ZEN 2011 (blue edition) software (Carl Zeiss) and edited with Adobe Photoshop CS3 Extended 10.0 (Adobe Systems; San Jose, CA, USA). Relative levels of immunofluorescent intensity were quantified using plugin algorithm software for ImageJ [National Institutes of Health (NIH); Bethesda, MD, USA]. Dendrite length was measured by tracing along individual neurons fluorescently labeled with MAP2 antibody using the broken line tool in ImageJ (Smith et al., 2009).

\section{Western Blotting}

Cell lysates were prepared in RIPA buffer supplemented with a mixture of protease and phosphatase inhibitors following $24 \mathrm{~h}$ treatment and separated by SDS-PAGE for immunoblotting. Primary antibodies used were anti-LAMP1 (catalog number NB120-19294) at a 1:100 dilution, anti-Beclin 1 (catalog number NB500-249) and anti-ATG5 (catalog number NB110-53818) at a 1:500 dilution, and anti-LC3B (catalog number NB600-1384) and anti-p62/SQSTM1 (catalog number NBP1-48320) at a 1:1000 dilution from Novus Biologicals (Littleton, CO, USA); anti-APG7/ATG7 (Santa Cruz Biotechnology; Santa Cruz, CA, USA; catalog number sc-33211) at a 1:50 dilution; and anti-GAPDH (Sigma-Aldrich; St. Louis, MO, USA; catalog number G9545) at a 1:1000 dilution. Primary antibodies were followed by incubation a secondary antibody conjugated to horseradish peroxidase (Cell Signaling Technology; Danvers, MA, USA; catalog number 7074) used at a 1:1000 dilution. Immunoblots were exposed to SuperSignal West Femto Substrate (Thermo Scientific; Waltham, MA, USA) and visualized using a ChemiDoc imaging system (Bio-Rad; Hercules, CA, USA).

\section{Statistics}

Data were analyzed by one-way ANOVA followed with Student Neuman-Keuls post hoc test for multiple comparisons using GraphPad Prism 5 (GraphPad Software; La Jolla, CA, USA). A value of $p<0.05$ was considered significant. Outliers were removed from the qRT-PCR analysis and identified with Grubbs' test using the QuickCalc outlier calculator (GraphPad Software), setting the alpha level to 0.05 .

\section{Results}

\section{Differential Expression of Autophagy Associated Genes in HIV-Infected Subjects with Two Types of Neurocognitive Impairment}

To begin to determine which autophagy associated genes might play a role in HIV-infected subjects with varying levels of NCI, qRT-PCR was used to examine differences in mRNA expression of common genes involved in various stages of the autophagy pathway (BECN1, MAP1LC3B, ATG7, and ATG5) and those marking autophagic activity (SQSTM1 and LAMP1) from post-mortem brain tissues of the four subject groups [HIV-negative (Group A), HIV-positive (Group B), HIV-positive with NCI (Group C), and HIV-positive with combined NCI and HIVE; Group D)] included in the NNTC Gene Array Project (Gelman et al., 2012). It should be noted that although the Gene Array Project describes samples collected from three brain regions (the frontal lobe white matter, frontal cortex, and basal ganglia), the tissue sample subset that we obtained to analyze consisted almost exclusively of samples from the frontal lobe white matter and frontal cortex (Table 1). Coincidently, many of the HIV-infected Gene Array Project subjects had reported histories of polysubstance abuse/dependence (Table 1), suggesting that our findings may, in part, be reflective of drugusing populations of infected individuals who could have altered cellular responses from interactive HIV-drug effects occurring in the CNS compared to infected non-substance users (Hauser et al., 2007). Although we did not detect any differences for BECN1 and MAP1LC3B (Figures 1A,B), and also for ATG12 (data not shown), we were able to detect significant differences in ATG7, ATG5, SQSTM1, and LAMP1 expression levels among the subject groups (Figures 1C-F). ATG7 levels were significantly higher in NCI-HIVE subjects compared to all the other groups while ATG5 levels were significantly lower in HIV-infected subjects with NCI compared to infected subjects without neurocognitive deficits. On the other hand, SQSTM1 levels had multiple significant differences between the various subject groups including lower expression in $\mathrm{HIV}$-infected subjects that were impaired without HIVE compared to higher expression in NCI-HIVE. Also, $L A M P 1$ expression levels were significantly higher in HIVinfected subjects that were unimpaired compared to uninfected subjects. Overall, these results suggest that at the transcriptional level while some autophagy-related genes may show differences 


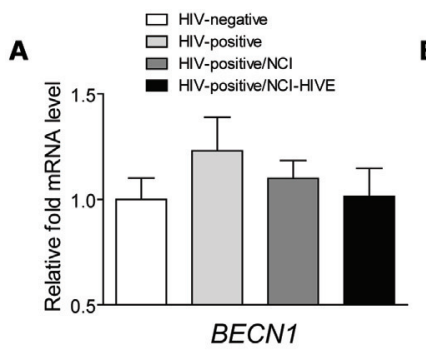

D

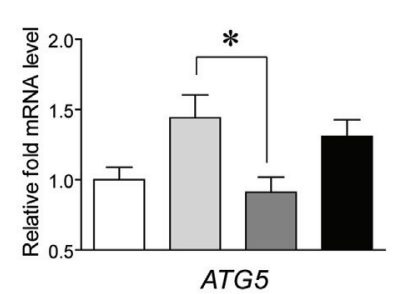

B

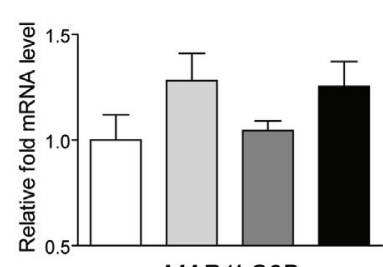

MAP1LC3B

E

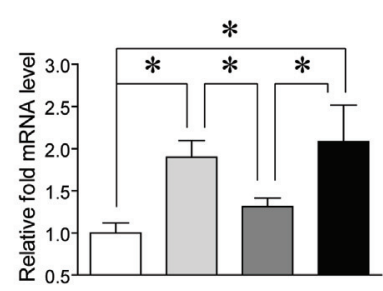

SQSTM1
C

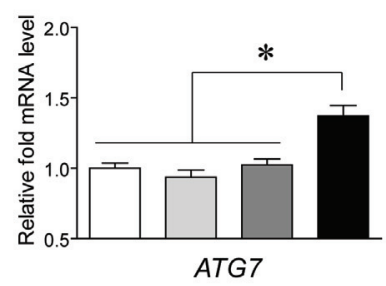

$\mathbf{F}$

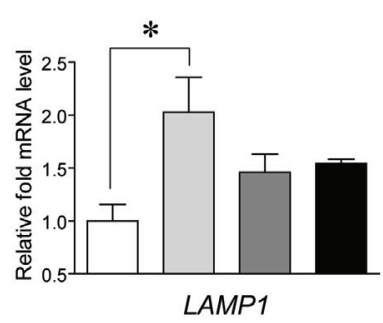

FIGURE 1 | Expression of mRNAs from autophagy associated genes in HIV-infected subjects with varying levels of neurocognitive impairment. Expression levels of the autophagy genes (A) BECN1, (B) MAP1LC3B, (C) ATG7, and (D) ATG5, and autophagic activity markers (E) SQSTM1 and (F) LAMP1 were measured by QRT-PCR across the indicated subject groups.
Data are presented relative to HIV-negative subjects which was set to a value of 1. BECN1: $F(3,24)=0.7201, p=0.5498 ; M A P 1 L C 3 B: F(3,23)=1.974$, $p=0.1461 ;$ ATG7: $F(3,24)=11.97, p=<0.0001 ;$ ATG5: $F(3,22)=4.350$, $p=0.0150 ;$ SQSTM1: $F(3,23)=4.958, p=0.0085 ; \operatorname{LAMP1}: F(3,24)=3.429$, $p=0.0331 ;{ }^{*} p<0.05$. Error bars show the SEM. in expression at various states of HIV infection, the level of autophagic activity may be lower during NCI-HIVE in the frontal lobe white matter and frontal cortex regions of the brain while mixed signaling of activity may occur during HIV infection leading up to NCI.

\section{Expression Patterns of Autophagy Associated Proteins in HIV-Infected Brain Tissue}

To determine whether there might be differences in protein expression of autophagy associated factors in relation to the qRT-PCR data, we performed immunohistochemistry on representative tissue that was from the same subject samples used in the PCR analysis. White matter sections were used due to the limited amounts of samples available from other brain regions. Autophagy associated protein expression was examined in Iba1-immunolabeled microglia to address whether the differences that we observed might be contributing to microglial-mediated neuroinflammation. Autophagy proteins and related markers were found to co-localize mostly with Iba1positive microglial cells but were also observed in Iba1-negative cell types (Figure 2A). Also, more Iba1-immunoreactive cells were observed in sections from subjects with NCI compared to the unimpaired groups (Figure 2A; arrow heads). However, when the overall immunofluorescent intensity was quantified, Iba1 expression levels were found to be higher in the NCI group without HIVE (Figure 2B). Furthermore, within the Iba1positive cells from the NCI groups, autophagy associated protein immunoreactivity on individual cells appeared to be higher (Figure 2A). When the overall immunofluorescent intensities were quantified for individual autophagy associated proteins, we were able to find significant differences in Beclin 1, APG7/ATG7,
ATG5, p62/SQSTM1, and LAMP1 expression levels among the subject groups (Figure 2C). Although HIV infection resulted in generally higher expression of LAMP1, and also for Beclin 1 with lower expression in the NCI-HIVE group compared to the other infected groups, higher expression in only the impaired groups was found for APG7/ATG7, ATG5, and p62/SQSTM1. While ATG5 expression was higher only in the NCI-HIVE group, the highest relative levels of expression among the subject groups were found for APG7/ATG7 in the NCI group without HIVE and p62/SQSTM1 in the NCI-HIVE group. Interestingly, more focal rather than diffuse patterns of immunoreactivity were observed among the proteins in the NCI-HIVE group (Figure 2A; arrows). Although all of these observations did not necessarily correlate with what we detected at the mRNA level, possibly due to post-translational modifications/stability and/or differences occuring specifically in the frontal lobe white matter and/or microglial cell type we examined, these results suggest that at the protein level while higher immunoreactivity may occur in the context of HIV infection and NCI, NCI-HIVE results in the accumulation of autophagy associated proteins in microglial cellular inclusion bodies which could be indicative of altered protein turnover and disruptions to autophagic flux in this cell type and is consistent with what we found for SQSTM1 by qRT-PCR.

\section{Examination of Autophagy Associated Genes Differentially Expressed in Various Brain Regions of HIV-Infected Subjects from Microarray Data}

Having assayed the subset of brain tissue samples that we had acquired for differences in autophagy associated factors at 


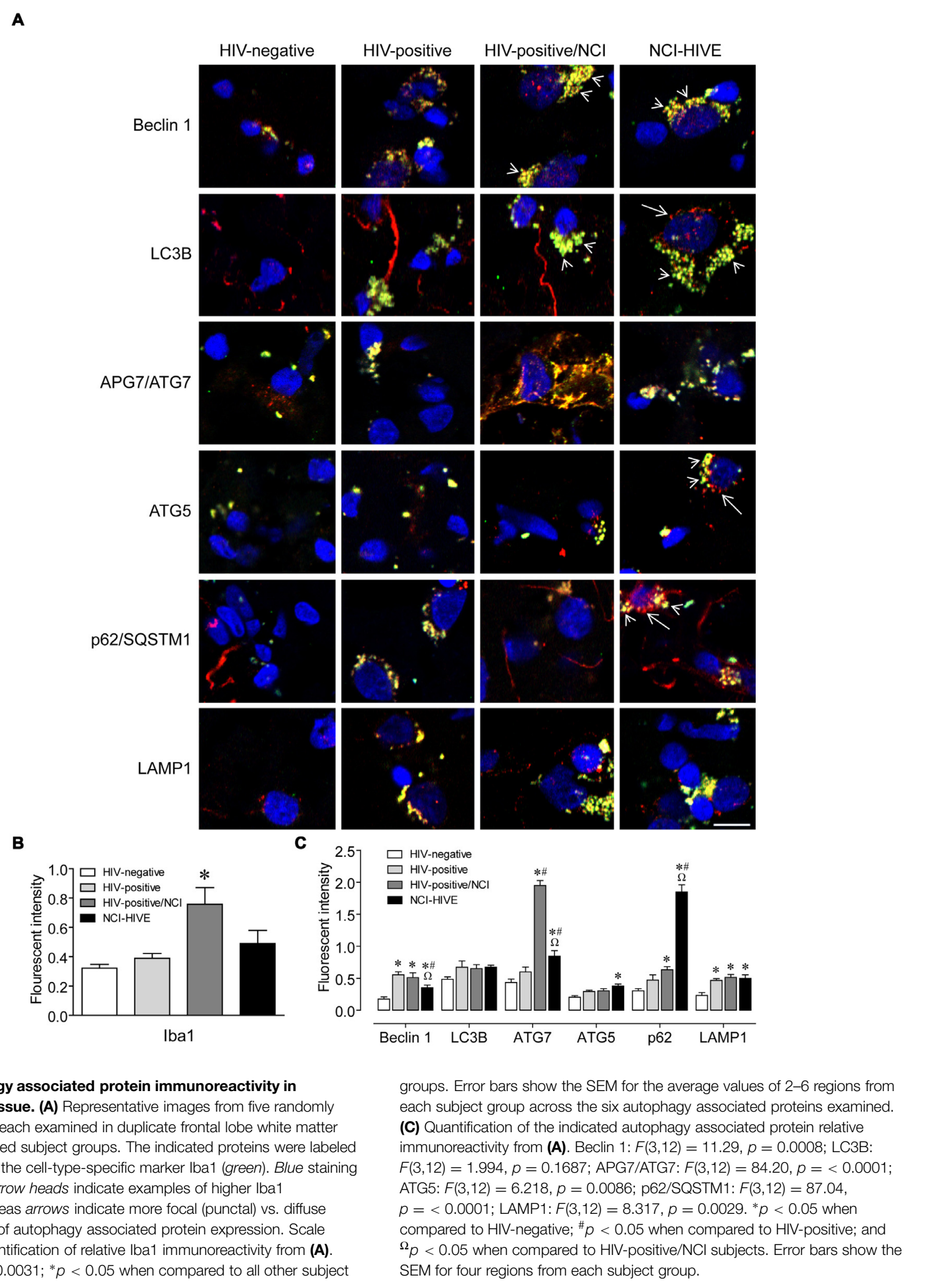

the mRNA and protein levels by standard methods, we next examined if expression of the genes that were measured by qRT-PCR, as well as whether other autophagy associated genes, might differ among the subject groups in a brain region-specific manner using microarray data that was retrieved and re-analyzed from the same set of subjects used in our study (Gelman et al., 
2012; Dever et al., 2014). Microarray data was used for this analysis as all of the tissue samples from individual brain regions included in the Gene Array Project for each subject were not available for qRT-PCR validation experiments, particularly those from the basal ganglia (Table 1). Heat maps were generated to display the expression pattern within individual probesets for the genes examined by qRT-PCR across the subject groups from the three brain regions where samples were collected: the frontal lobe white matter, frontal cortex, and basal ganglia (Figures 3A-C), and we used a multi-class linear models for microarray data (Limma; parametric) analysis of the microarray data to access differential expression between the subject groups (Smyth, 2004). Out of these genes, only LAMP1 levels were found to be statistically significant when comparing uninfected and NCI-HIVE subjects in the basal ganglia, which was detected with a particular probeset (201553_s_at; Figure 3D), as opposed to the higher levels in HIV-infected subjects without NCI that were detected using qRT-PCR with samples almost exclusively from the frontal lobe white matter and frontal cortex (Table 1). This result for $L A M P 1$ in the basal ganglia was also found in the original microarray analysis of this data set using localpooled-error (non-parametric) tests for statistical comparisons (Gelman et al., 2012). Although the microarray and qRT-PCR data did not correlate for the genes we had examined up to this point, most likely due to the particular tissue sample subset we used, at the transcriptional level the combined results suggest that autophagic processes may differ in a brain region-specific manner during HIV infection, more closely associated with the NCI-HIVE condition, with higher activity in the basal ganglia as indicated by LAMP1 levels from the microarray data and lower activity in the frontal lobe white matter and frontal cortex as indicated by SQSTM1 levels using qRT-PCR. Furthermore, the results from comparison of the genes and corresponding proteins suggest another level of complexity whereby differences in mRNA and protein expression may not necessarily correlate due to modifications/stability at the post-translational level which

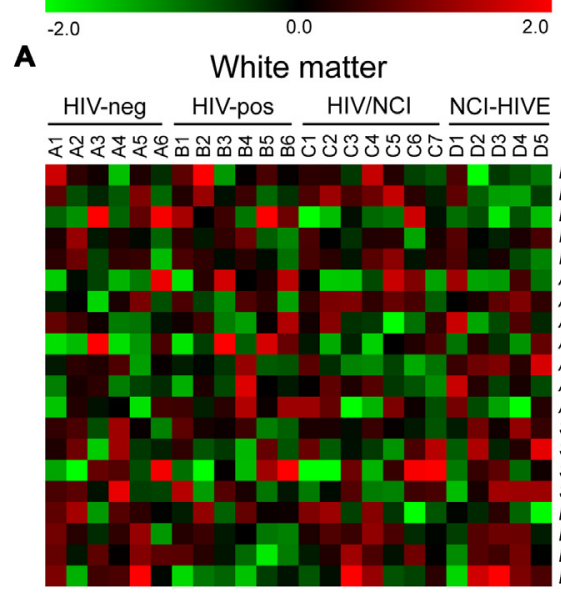

C

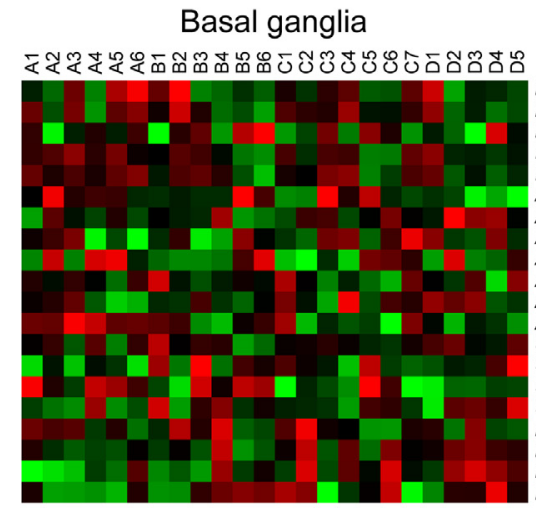
. BECN1 (208945_s_at) BECN1 (208945_s_at)
BECN1 (208946_s_at) BECN1 (156165 $\overline{2}$ at)
MAP1LC3B $(208785$ MAP1LC3B (208785_s_at) MAP1LC3B (208786_s_at) ATG7 (218673_s_at) ATG7 (222709_at) ATG7 (224025_s_at) ATG7 (1569827_at) ATG5 (202511_s_at) ATG5 (202512_s_at) ATG5 (210639s at) SQSTM1 (2014771_s_at) SQSTM1 (213112_s_at) SQSTM1 (239004_at) SQSTM1 (244804_at) LAMP1 (201551_s_at) LAMP1 (201552_at) LAMP1 (201553_s_at) LAMP1 (213728_at)

D

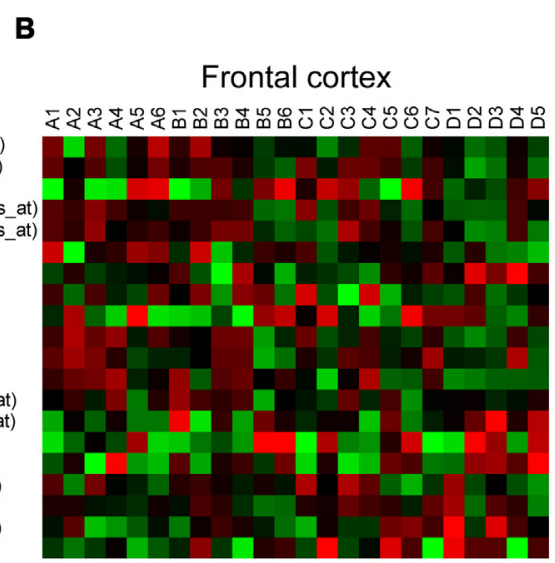
BECN1 (208945_s_at) BECN1 (208946_s_at) BECN1 (1561652 -at) MAP1LC3B (208785_s_at) MAP1LC3B (208786_s_at) ATG7 (218673_s_at) ATG7 (222709_at) ATG7 (224025_s_at) ATG7 (1569827_at) ATG5 (202511_s_at) ATG5 (202512_s_at) ATG5 (210639s at) SQSTM1 (201471]_s_at) SQSTM1 (213112_s_at) SQSTM1 (239004_at) SQSTM1 (244804_at) LAMP1 (201551_s_at) LAMP1 (201552 at) LAMP1 (201553_s at) LAMP1 (213728-at)

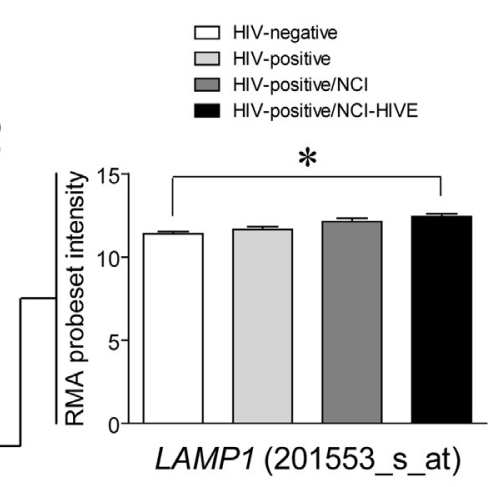

FIGURE 3| Autophagy associated gene expression from microarrays in three brain regions of HIV-infected subjects. Heat maps are shown for the indicated genes from (A) frontal lobe white matter, (B) frontal cortex, and (C) basal ganglia of subjects that were HIV-negative (A1-A6), HIV-positive (B1-B6), HIV-positive with neurocognitive impairment ( $\mathrm{NCl}$; $\mathrm{C} 1-\mathrm{C} 7)$, and $\mathrm{HIV}$-positive with combined $\mathrm{NCl}$ and HIV encephalitis (HIVE; D1-D5). IDs of individual probesets included in the array for each gene are given in parenthesis. (D) A significant difference of RMA probeset intensity values from the microarray data analyses was found in the basal ganglia for LAMP1 (201553_s_at) between HIV-negative (Group A) and NCI-HIVE (Group D) subjects; ${ }^{*} p<0.05$. Error bars show the SEM. 
may also be occurring a brain region- and/or cell-type-specific manner.

In addition to the genes examined thus far, we also wanted to determine whether our Limma analysis found differences with probesets for other autophagy associated genes as well. The analysis found that the autophagy-related gene $A T G 4 D$, as well as other genes involved in autophagy including DRAM1 and KIAA0226, had significantly differential expression between uninfected and NCI-HIVE subjects in the basal ganglia, and ATG12 and ATG13 as well as CTSB, TM9SF1, and WIPI1 levels significantly differed when all the subject groups were compared in this same brain region, although no significant pairwise differences were found between any two particular groups (data not shown). Also, ATG3 and ATG4D as well as C12orf44 (ATG101), AMBRA1, DRAM1, KIAA0226, RAB24, and ULK1 in the frontal lobe white matter and DAP and DRAM1 in the frontal cortex were found to have significant differences across all groups (data not shown). The differential expression of these other genes that we did not examine by qRT-PCR might represent interesting targets for future validation studies on the differences that occur in autophagy during various states of HIV infection, and, in particular, NCI-HIVE. Furthermore, these results suggest that the basal ganglia, where most of the microarray differences were found, may be a key brain region to evaluate the impact of differing autophagic processes on the neurocognitive function of HIV-infected patients.

\section{Effect of Supernatant from HIV-1-Infected Microglia on Neuronal Autophagic Activity in the Presence of Morphine}

As disruptions to the autophagic activity of primary rodent neurons have been reported to result with treatment of supernatant from SIV-infected microglia cultured ex vivo (Alirezaei et al., 2008), and HIV-1-infected primary human microglia exposed to morphine in vitro can affect autophagy which may influence the downstream neurotoxic activities of this cell type (El-Hage et al., 2015), we next tested whether in vitro exposure of primary human neurons to supernatant from HIV-1-infected primary human microglia in the presence of morphine might also modify autophagic processes in a manner that could result in altered neuronal function leading to NCI. Although many of the HIV-infected Gene Array Project subjects had substance use histories with various drugs of abuse (Table 1), we chose to examine the interactive effects of HIV and morphine on neurons as opiates with abuse liability that preferentially activate the $\mu$-opioid receptor (MOR) such as morphine can exacerbate the neuropathogenesis of HIV by mechanisms which are not fully understood (Hauser et al., 2005). However, it is generally believed glia play a central role in mediating opiate drug-HIV interactions on neurons through bystander events via neurotoxic signaling molecules and viral proteins released from infected and activated cells such as microglia and that opiates such as morphine can potentiate these effects (Hauser et al., 2012). Interestingly, while the supernatant from HIV-1-infected microglia alone did not result in differences to autophagic activity as determined by measuring p62/SQSTM1 immunoreactivity in exposed neurons, supernatant from infected microglia in the presence of morphine resulted in significantly higher p62/SQSTM1 expression (Figures 4A,B), suggesting inhibition of autophagy as we found for NCI-HIVE subjects in vivo. In support of these observations, when wholecell lysates were examined by western blotting analysis, we found that p62/SQSTM1 expression levels were highest for infected supernatant and combined morphine treatment compared to the other supernatant treatments, and which corresponded with concomitantly lower LAMP1 levels (Figure 4C). Also, when the length of neuronal dendrites was examined under these same conditions, we observed that morphine alone was able to reduce dendritic length which was further enhanced in the combined presence of supernatant from uninfected microglia, and even more so in combination with supernatant from HIV-1-infected microglia, demonstrating an additive trend in response to the supernatant and morphine treatments (Figure 4D). These results suggest that differences in dendritic length can occur in response to morphine and HIV-1-infected microglial supernatant alone rather than as a consequence of inhibited autophagic activity which was only observed with co-treatment. Furthermore, anatomical variations might occur in a transient/reversible manner to initial exposure possibly resulting in milder forms of NCI, while severe NCI may correlate with structural differences coupled to mechanistic alterations in cellular function through inhibition of autophagic activity that could lead to more permanent neurocognitive deficits and dementia as with NCIHIVE subjects.

\section{Influence of HIV-1 Tat and Morphine Treatment on Autophagy in Neurons}

Since the HIV-1 Tat protein can mediate neuronal toxicity which can be enhanced in the combined presence of morphine (Zou et al., 2011), we then tested if treatment of primary human neurons with HIV-1 Tat and morphine would have similar effects to what we found with the supernatant from HIV-1-infected microglia and morphine to further delineate whether various cellular-oriented factors such as inflammatory molecules released by infected microglia or viral proteins shed from these cells may be responsible for the interactive effects by first using a fluorescent reporter system to monitor autophagic flux. Neurons were transfected with a plasmid encoding a mRFP-GFP-LC3 tandem fluorescently-tagged LC3 reporter with which green (GFP) and yellow (GFP + mRFP) fluorescence are observed prior to the fusion of autophagosomes with lysosomes whereas only red (mRFP) fluorescence is present in post-fusion autolysosomes (Kimura et al., 2007). Following transfection, neurons were treated with HIV-1 Tat [an initial concentration of $100 \mathrm{nM}$ was tested which can elicit functional deficits in neurons and glia similar to those occurring in HIV infection and is considered to reflect levels seen pathophysiologically (Nath et al., 1999; Singh et al., 2004; El-Hage et al., 2005, 2008)] and morphine [500 $\mathrm{nM}$ was chosen for the ability of this concentration to fully activate MOR and synergistically enhance Tat-mediated neurotoxicity (Gurwell et al., 2001; Zou et al., 2011)] alone or in combination for $8 \mathrm{~h}$ and the differential patterns of fluorescence were observed (Figure 5A). When the number of red fluorescent signals (puncta) occurring in autolysosomes were 
A

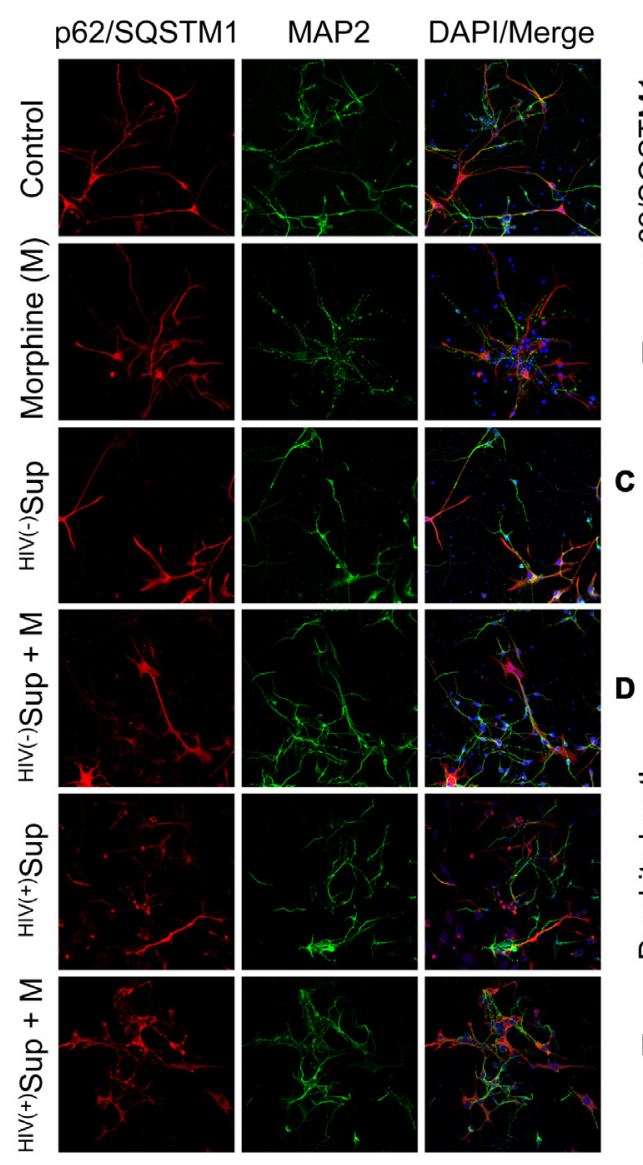

B

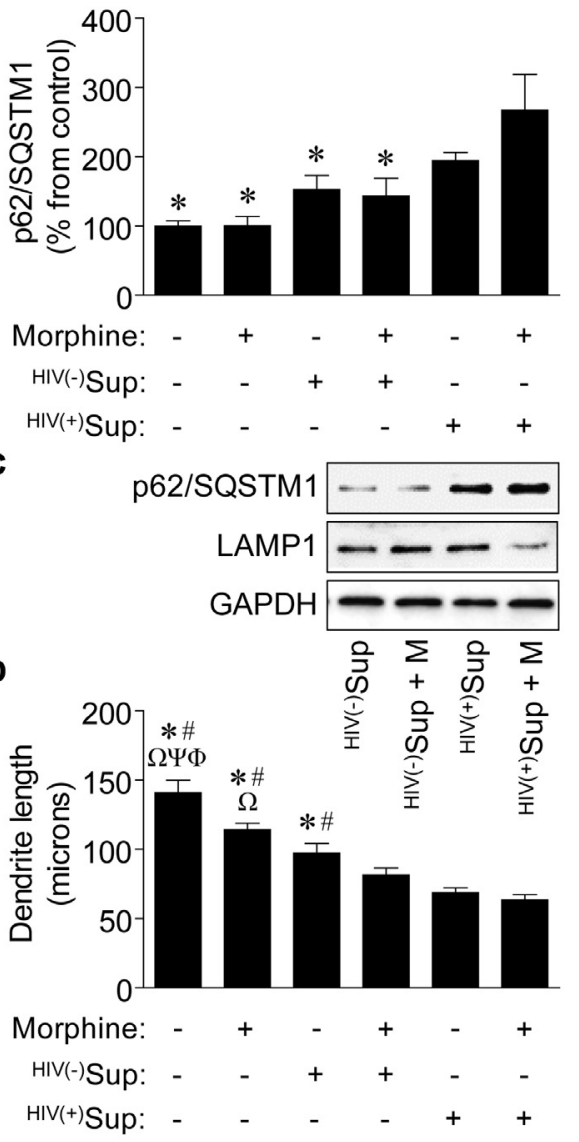

FIGURE 4 | Effects on autophagic activity and dendritic length of neurons exposed to supernatant from HIV-1-infected microglia in combination with morphine. (A) Representative images of neurons with the indicated treatments. Sup, supernatant from uninfected $[H I V(-)]$ and HIV-1-infected $[H I V(+)]$ microglia. Cells were immunolabeled with antibodies to the autophagic activity marker p62/SQSTM1 (red) and the neuronal cell-type-specific marker MAP2 (green). DAPI (b/ue) staining indicates cell nuclei. (B) Quantification of p62/SQSTM1 immunoreactivity from (A). Data are presented as the percentage of control cells which was set at 100; $F(5,24)=5.882, p=0.0011 ;{ }^{*} p<0.05$ when compared to ${ }^{H I V}(+)$ Sup + morphine treatment. (C) Western blotting analysis of p62/SQSTM1 and LAMP1 expression levels for the indicated treatments. GAPDH was used as a loading control. Blots are representative of three independent experiments. (D) Measurement of dendrite length from (A). $F(5,24)=26.15, p=<0.0001 ;{ }^{\Phi} p<0.05$ when compared to morphine; ${ }^{\Psi} p<0.05$ when compared to HIV(-) Sup; ${ }^{\Omega} p<0.05$ when compared to $\operatorname{HIV}(-)$ Sup + morphine; ${ }^{\#} p<0.05$ when compared to $\operatorname{HIV}(+)$ Sup; and $* p<0.05$ when compared to $\operatorname{HIV}(+)$ Sup + morphine treatment. Error bars show the SEM for five randomly selected fields totaling at least 100 cells from each group. manually quantified, we found that HIV-1 Tat and morphine treatment in combination resulted in significantly lower numbers of autophagosomal-lysosomal fusion events compared to all the other treatments (Figure 5B). However, when whole-cell lysates were next examined by western blotting analysis for expression levels of various autophagy associated proteins at a later time point $(24 \mathrm{~h})$, autophagic activity appeared to be higher. While LC3B levels remained relatively constant across the treatment conditions and LAMP1 levels were higher for a lower concentration of Tat $(1 \mathrm{nM})$ which was sustained using higher concentrations of Tat $(10$ and $100 \mathrm{nM})$ in the presence of morphine, Beclin 1, APG7/ATG7, and ATG5 exhibited higher levels of expression coinciding with combined Tat and morphine exposure, that were mostly associated with the highest concentration of Tat used (100 nM), when p62/SQSTM1 levels were concomitantly lower (Figure 5C). We also observed that dendritic beading, which is indicative of excitotoxic stress and/or injury (Ellis et al., 2007), was significantly higher with HIV-1 Tat treatment and Tat treatment in combination with morphine compared to control cells (Figure 5D). All combined, our in vitro results suggest that while HIV infection can result in bystander effects on neuronal morphology, disruptions to specific cellular mechanisms such as autophagy may occur more readily from the interactive effects of HIV and associated viral proteins with substances of abuse/dependence such as morphine. Furthermore, when morphine is present, the consequences of neurotoxic and inflammatory factors released by infected cells on neuronal autophagic processes may be different than that of viral proteins which could in themselves have differing effects in a timedependent manner. 


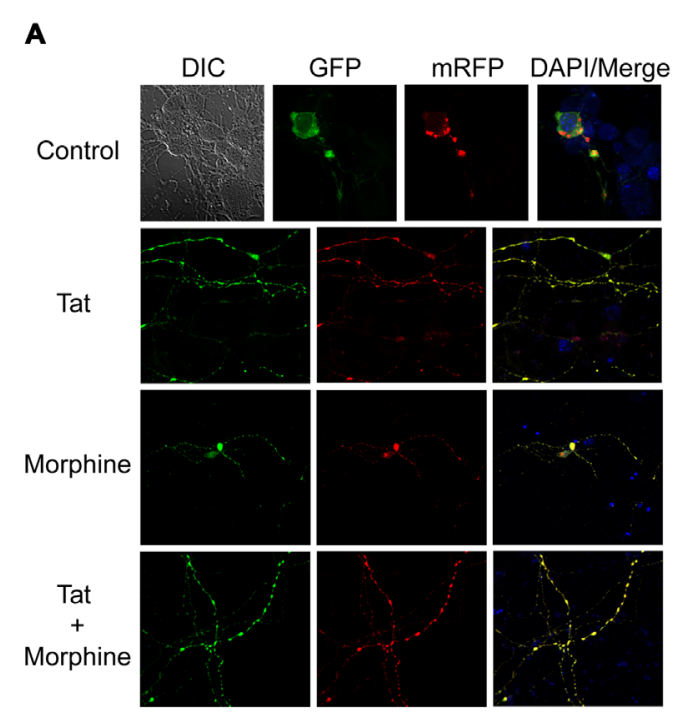

$\mathbf{B}$

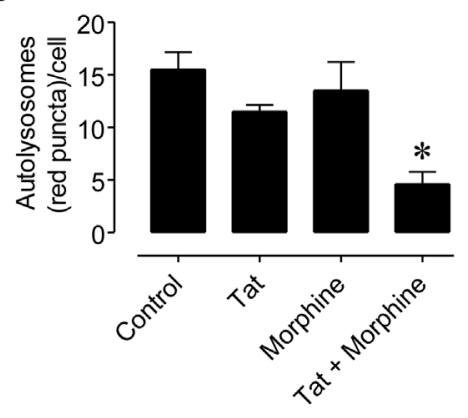

FIGURE 5 | Differences in neuronal autophagy and dendrite varicosity following HIV-1 Tat protein and morphine treatment.

(A) Representative images of neurons transfected with a fluorescent reporter plasmid to monitor autophagic flux at $8 \mathrm{~h}$ following the indicated treatments. GFP (green) and GFP + mRFP (yellow) fluorescence are observed prior to the fusion of autophagosomes with lysosomes whereas only mRFP (red) fluorescence is present in post-fusion autolysosomes. DIC, differential interference contrast microscopy image. DAPI (b/ue) staining
C

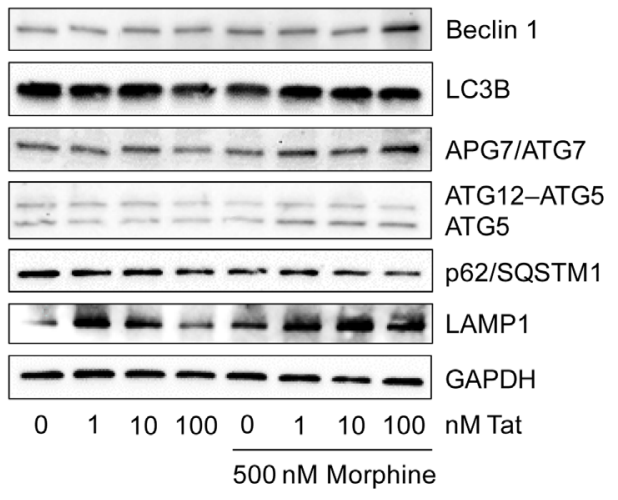

D

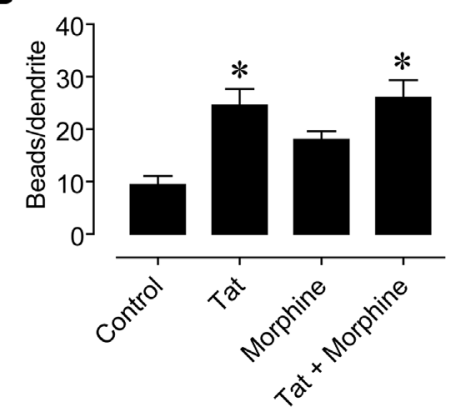

indicates cell nuclei. (B) Quantification of autolysosomes (red puncta) from (A). $F(3,13)=8.756, p=0.0019 ;{ }^{*} p<0.05$ when compared to all other groups. (C) Western blotting analysis of the indicated autophagy associated protein levels at $24 \mathrm{~h}$ following the indicated treatments. GAPDH was used as a loading control. Blots are representative of three independent experiments. (D) Quantification of dendrite beading from (A) $F(3,77)=6.429, p=0.0006 ;{ }^{*} p<0.05$ when compared to control cells. Error bars show the SEM.

\section{Discussion}

In the present study, we investigated the role of autophagy in the brains of HIV-infected subjects with NCI with and without combined HIVE. These findings are unique in that our subject group categories allowed us to determine whether the differences we detected in autophagy may be more associated with encephalitis than NCI. For instance, this seemed to be the case when we examined SQSTM1 expression using qRT-PCR, and our results were in agreement with a previous report that found higher mRNA levels of this gene in the frontal lobe of SIV encephalitic (SIVE) monkeys and frontal cortex of human subjects with HAD (Alirezaei et al., 2008). It should be noted that most of our subjects with NCI had neurocognitive diagnoses of MCMD and possible HAD, while those with NCI-HIVE were diagnosed with HAD and probable HAD (Table 1).

We began our search for differences in autophagy associated factors by performing qRT-PCR and immunohistochemical analyses on brain tissue samples obtained from various categories of HIV-infected subjects, followed by examining microarray data generated from these same subjects within three brain regions. Interestingly, out of several genes commonly examined in autophagy studies that we also investigated, the microarray data only revealed a significant difference in expression for the autophagic activity marker LAMP1 which was detected with a particular probeset in the basal ganglia. However, we were able to detect significant expression differences among the subject groups for other genes involved in autophagy as well using qRT-PCR. In addition, the difference found for $L A M P 1$ in the microarray data was between uninfected and NCIHIVE subjects whereas the difference detected by qRT-PCR was between uninfected and HIV-infected subjects without NCI. The discrepancy in these results could be at least partially explained by the particular tissue sample subset provided to us which consisted almost exclusively of samples from the frontal lobe white matter and frontal cortex (Table 1), as all of the samples 
used in the arrays were not available. This limitation points to the importance and need for brain banking and availability of wellmatched samples from different anatomical regions and disease state categories of HIV-infected subjects for future validation studies.

Furthermore, we found differences with our immunohistochemical analysis of autophagy associated proteins in microglia that were not reflected in the qRTPCR data suggesting differential regulation at the transcriptional, translational, and post-translational levels. For example, the immunoreactivity of proteins that we examined was generally higher in the impaired groups. However, our results for p62/SQSTM1 were fairly consistent by both qRT-PCR and immunohistochemistry with higher expression in the NCI-HIVE group. Overall, our findings of immunoreactivity were mostly in agreement with a previous report that found higher expression levels for autophagy associated proteins in the frontal cortex of HIVE individuals by western blotting analysis (Zhou et al., 2011). Interestingly, Zhou et al. (2011) reported that no differences in the protein expression levels were found between HIVE-positive individuals with and without HAD. Combined with our findings, the data again suggest that encephalitis more than NCI alone may be responsible for some of the observed differences. Although we were able to observe differences in immunoreactivity using tissue sections, we were unable to perform other assays to confirm these findings due to the limited amount of tissue available. The availability of larger tissue sample sizes from the categories of subjects that we examined will be useful to further evaluate our findings at the protein level by more quantitative methods such as western blotting.

In addition to differences in immunoreactivity, we also found qualitative differences in the expression of autophagy associated proteins with more focal rather than diffuse patterns in microglia from the NCI-HIVE group. This finding suggests that in the HIVE condition, accumulation of proteins associated with autophagy in cellular inclusion bodies may be indicative of altered protein turnover, which could be related to what we observed as higher levels of immunoreactivity, and is manifested by disruptions in autophagic activity/flux. Our results are supported by a previous report that found more focal patterns of p62/SQSTM1 expression in the hippocampus of monkeys with SIVE and HIV-infected individuals with HAD (Alirezaei et al., 2008). Indeed, such formations have been described in patients with a variety of neurodegenerative diseases (Zatloukal et al., 2002). Furthermore, SQSTM1 transcription can be higher as part of inclusion body formation (Nakaso et al., 2004), which supports our qualitative and quantitative (p62/SQSTM1) immunohistochemical results with the qRTPCR data for this gene that we detected in the NCI-HIVE group. Overall, our findings with the human brain tissues suggest HIVE, rather than NCI alone, is more associated with differences to autophagic processes in microglia which may then through altered intercellular transmission of released factors result in similar differences within neuronal cells that could lead to neurodegeneration, and p62/SQSTM1 is a responsive marker in this regard. Future in vitro studies will be needed to systematically explore the connections between disrupted autophagy in microglia and the implications for autophagic and other cellular processes in bystander cell types such as neurons.

While our human brain tissue data was fairly consistent with the findings of both Alirezaei et al. (2008) and Zhou et al. (2011), the combined results from these and our study seem somewhat contradictory suggesting that autophagy levels are both higher and lower during HAD/HIVE. However, it may be possible to reconcile these findings in that induced or suppressed autophagic activity in response to prolonged HIV infection to, for example, clear toxic factors and affect viral replication, followed by compensatory differences to offset these effects itself suggests a mechanism whereby mixed signals and dysregulation in an attempt for cell survival is manifested as cellular dysfunction which could lead to NCI. Interestingly, a more recent study found that non-progressor $\mathrm{HIV}$-infected patients have higher autophagy compared to normal progressors when peripheral blood mononuclear cells (PBMCs) were examined (Nardacci et al., 2014). This finding suggests that, at least in the periphery, higher autophagy levels may be critical for controlling the initial spread of HIV infection to the CNS, preventing the formation of viral reservoirs in the brain which could eventually lead to HAD and HIVE. Future studies will be needed to evaluate the connections between the higher and lower autophagic activity to identify targets which could possibly be used to offset and/or coordinate the opposing events.

In conjunction with our human in vivo data, we also found that morphine can interact with the supernatant from HIV-1infected microglia and HIV-1 Tat protein in opposing ways to cause differences of neuronal autophagy in vitro. These results suggest that opioid exposure may uniquely activate some of the in vivo effects of HIV through modulation of the autophagic pathway in this cell type which could accelerate NCI compared to bystander events from HIV infection alone. Furthermore, these mechanistic differences resulting from interactive effects could potentially result in more permanent alterations to cellular function compared to the morphological differences that we observed in dendritic length and beading, which may be reversible (Ellis et al., 2007), with individual treatments. However, as a common route of HIV infection in substance-abusing individuals is following injection drug use (Leshner, 1998), the consequences of preexisting and irreversible dendrite differences from chronic drug exposure that can itself result in NCI may be exacerbated upon the onset and duration of HIV infection with more additive rather than interactive effects in this sequence of events leading to further neuronal injury and death.

Interestingly, while our in vivo human brain tissue data correlated with that of both Alirezaei et al. (2008) and Zhou et al. (2011), our in vitro results did not as a significant effect of supernatant from HIV-1-infected primary human microglia and Tat treatment alone on autophagy in primary human neurons was not found for the most part. Alirezaei et al. (2008) demonstrated that supernatant from SIV-infected microglia cultured ex vivo inhibited autophagy in primary rodent neurons, whereas Zhou et al. (2011) showed HIV-1 gp120 protein exposure resulted in higher autophagic activity of SK-N-SH neuroblastoma cells. However, our results in conjunction with morphine on autophagy did agree with both Alirezaei et al. (2008) and 
Zhou et al. (2011) showing induction with viral protein and inhibition for infected microglial supernatant following $24 \mathrm{~h}$ of treatment, while the interactive effect with Tat appeared to be dynamic with lower autophagic activity at $8 \mathrm{~h}$ co-exposure. Although the effects on autophagy that we were able to find compared to Alirezaei et al. (2008) and Zhou et al. (2011) may have resulted from exacerbation by morphine and could also be attributed to variations in the species, SIV/HIV strains, HIV proteins, cell systems, etc. used between all three studies, future characterization of supernatant from infected microglial cells and testing the effect of individual components on autophagic processes in neurons will be necessary for understanding the underlying differences.

Furthermore, recently published in vitro data from our lab supports the in vivo findings of this study that showed HIV-1 infection can affect autophagy in primary human microglia and morphine can modulate these differences which could influence viral replication, cytokine/chemokine production and release, and potentially other factors involved in mediating neurotoxicity (El-Hage et al., 2015). Therefore, future evaluation of the in vitro effects from morphine and other drugs of abuse on autophagy in the context of HIV infection examining individual CNS cell types may provide new insights for particular therapeutics directed toward the autophagic pathway and which target an individual type of cell. However, at present, the interactions between HIV and autophagy are largely unknown in astrocytes (Dinkins et al., 2015), and other CNS cell types such as pericytes and brain microvascular endothelial cells involved in bloodbrain barrier integrity. Elucidation of the basic mechanisms by which HIV infection affects autophagy in these cells will be necessary before detailed studies on the impact of drugs in these processes can be systematically explored. As astrocytes are thought to be important for driving HIV-opiate interactions through intercellular feedback loops with microglia (Hauser et al., 2012), their role in autophagy during HIV infection may represent a potential therapeutic strategy targeting the bystander effects of glial responses on neurons and worth intense future investigation. In addition, detailed studies on a single drug may not be feasible using in vivo human samples, and particularly with our tissues, as many of the infected subjects from our study had a history of polysubstance abuse/dependence (Table 1). This constraint further underscores the notion that availability of tissue samples from HIV-infected subjects with well-documented patient histories of a particular substance use, as well as those with no reported use history, will be necessary for future evaluation when using in vivo human samples to examine an individual drug and also highlights the need for more in vitro studies on the interactive effects from exposure to multiple drugs of abuse in the context of HIV infection.

\section{Conclusion}

Our findings demonstrating differences of autophagy in microglial cells from NCI-HIVE subjects are one of the few reports that suggest microglia may represent a better upstream therapeutic target to modulate autophagy in the context of HIV infection to ameliorate neurodegeneration rather than neuronal cells themselves. However, for HIV-infected individuals exposed to morphine, direct neuronal targeting may be as important as targeting glia. Therefore, substance-abusing individuals may require more aggressive and multifaceted treatment regimens targeting the direct and indirect cellular causes of neurocomplications from HIV infection than non-substance users.

\section{Author Contributions}

$\mathrm{SD}$ and NE-H designed the study and analyzed and interpreted the experimental data. SD, MR, JL, and NE-H conducted the experiments. $\mathrm{BC}$ retrieved and analyzed the microarray data. SD drafted the manuscript and NE-H critically revised the work for important intellectual content. All authors approved the final version of the manuscript.

\section{Acknowledgments}

We thank Kurt F. Hauser for use of the human tissue samples that were analyzed in this study as well as for use of laboratory space and equipment from which part of the data in this manuscript were generated. We also thank Megan A. O’Brien for previous assistance with the microarray data retrieval and analysis, David A. Gewirtz for suggestions regarding the manuscript, and we are most grateful to the subjects who donated tissue. We gratefully acknowledge the support of the NIH-National Institute on Drug Abuse (NIDA) grants F32 DA033898 to SD and R01 DA036154 to NE-H. The human tissue provided by the NNTC for this publication was made possible from NIH funding through the National Institute of Mental Health (NIMH) and National Institute of Neurological Disorders and Stroke (NINDS) by the following grants: Manhattan HIV Brain Bank: U01 MH083501, R24 MH59724; Texas NeuroAIDS Research Center: U01 MH083507, R24 NS45491; National Neurological AIDS Bank: 5U01 MH083500, NS38841; California NeuroAIDS Tissue Network: U01 MH083506, R24 MH59745; and Statistics and Data Coordinating Center: U01 MH083545, N01 MH32002. Part of the microscopy in this study was performed at the Virginia Commonwealth University (VCU) Department of Anatomy and Neurobiology Microscopy Facility, supported, in part, with funding from NIH-NINDS Center core grant 5P30 NS047463. Tissue sectioning was performed by the VCU Massey Cancer Center Tissue and Data Acquisition and Analysis Core Facility, supported, in part, with funding from NIH-National Cancer Institute (NCI) Cancer Center core support grant P30 CA016059. This publication's contents are solely the responsibility of the authors and do not necessarily represent the official views of the NIH or the NNTC or endorsement by any other individuals. 


\section{References}

Alirezaei, M., Kiosses, W. B., Flynn, C. T., Brady, N. R., and Fox, H. S. (2008). Disruption of neuronal autophagy by infected microglia results in neurodegeneration. PLoS ONE 3:e2906. doi: 10.1371/journal.pone.0002906

Anderson, E., Zink, W., Xiong, H., and Gendelman, H. E. (2002). HIV-1associated dementia: a metabolic encephalopathy perpetrated by virus-infected and immune-competent mononuclear phagocytes. J. Acquir. Immune Defic. Syndr. 31(Suppl. 2), S43-S54. doi: 10.1097/00126334-200210012-00004

Antinori, A., Arendt, G., Becker, J. T., Brew, B. J., Byrd, D. A. Cherner, M., et al. (2007). Updated research nosology for HIV-associated neurocognitive disorders. Neurology 69, 1789-1799. doi: 10.1212/01.WNL. $0000287431.88658 .8 \mathrm{~b}$

Benjamini, Y., and Hochberg, Y. (1995). Controlling the false discovery rate: a practical and powerful approach to multiple testing. J. R. Stat. Soc. 57, 289-300. doi: $10.2307 / 2346101$

Bjorkoy, G., Lamark, T., Brech, A., Outzen, H., Perander, M., Overvatn, A., et al. (2005). p62/SQSTM1 forms protein aggregates degraded by autophagy and has a protective effect on huntingtin-induced cell death. J. Cell Biol. 171, 603-614. doi: $10.1083 /$ jcb. 200507002

Cheng-Mayer, C., and Levy, J. A. (1988). Distinct biological and serological properties of human immunodeficiency viruses from the brain. Ann. Neurol. 23(Suppl.), S58-S61. doi: 10.1002/ana.410230716

Cherra, S. J. III, and Chu, C. T. (2008). Autophagy in neuroprotection and neurodegeneration: a question of balance. Future Neurol. 3, 309-323. doi 10.2217/14796708.3.3.309

Dever, S. M., Costin, B. N., Xu, R., El-Hage, N., Balinang, J., Samoshkin, A., et al. (2014). Differential expression of the alternatively spliced OPRM1 isoform mu-opioid receptor-1K in HIV-infected individuals. AIDS 28, 19-30. doi: 10.1097/QAD.0000000000000113

Dever, S. M., Xu, R., Fitting, S., Knapp, P. E., and Hauser, K. F. (2012). Differential expression and HIV-1 regulation of mu-opioid receptor splice variants across human central nervous system cell types. J. Neurovirol. 18, 181-190. doi 10.1007/s13365-012-0096-Z

Dinkins, C., Pilli, M., and Kehrl, J. H. (2015). Roles of autophagy in HIV infection. Immunol. Cell Biol. 93, 11-17. doi: 10.1038/icb.2014.88

El-Hage, N., Bruce-Keller, A. J., Yakovleva, T., Bazov, I., Bakalkin, G., Knapp, P. E., et al. (2008). Morphine exacerbates HIV-1 Tat-induced cytokine production in astrocytes through convergent effects on $\left[\mathrm{Ca}^{2+}\right] \mathrm{i}, \mathrm{NF}-k a p p a B$ trafficking and transcription. PLoS ONE 3:e4093. doi: 10.1371/journal.pone.0004093

El-Hage, N., Dever, S. M., Podhaizer, E. M., Arnatt, C. K., Zhang, Y., and Hauser K. F. (2013). A novel bivalent HIV-1 entry inhibitor reveals fundamental differences in CCR5-mu-opioid receptor interactions between human astroglia and microglia. AIDS 27, 2181-2190. doi: 10.1097/QAD.0b013e32836 39804

El-Hage, N., Gurwell, J. A., Singh, I. N., Knapp, P. E., Nath, A., and Hauser, K. F. (2005). Synergistic increases in intracellular $\mathrm{Ca}^{2+}$, and the release of MCP-1, RANTES, and IL- 6 by astrocytes treated with opiates and HIV-1 Tat. Glia 50 91-106. doi: 10.1002/glia.20148

El-Hage, N., Rodriguez, M., Dever, S. M., Masvekar, R. R., Gewirtz, D. A., and Shacka, J. J. (2015). HIV-1 and morphine regulation of autophagy in microglia: limited interactions in the context of HIV-1 infection and opioid abuse. J. Virol. 89, 1024-1035. doi: 10.1128/JVI.02022-14

El-Hage, N., Rodriguez, M., Podhaizer, E. M., Zou, S., Dever, S. M., Snider, S. E., et al. (2014). Ibudilast (AV411), and its AV1013 analog, reduce HIV1 replication and neuronal death induced by HIV-1 and morphine. AIDS 28 1409-1419. doi: 10.1097/QAD.0000000000000291

Ellis, R., Langford, D., and Masliah, E. (2007). HIV and antiretroviral therapy in the brain: neuronal injury and repair. Nat. Rev. Neurosci. 8, 33-44. doi: $10.1038 / \mathrm{nrn} 2040$

Espert, L., Varbanov, M., Robert-Hebmann, V., Sagnier, S., Robbins, I., Sanchez, F., et al. (2009). Differential role of autophagy in CD4 T cells and macrophages during X4 and R5 HIV-1 infection. PLoS ONE 4:e5787. doi: 10.1371/journal.pone.0005787

Garden, G. A. (2002). Microglia in human immunodeficiency virus-associated neurodegeneration. Glia 40, 240-251. doi: 10.1002/glia.10155

Gelman, B. B., Chen, T., Lisinicchia, J. G., Soukup, V. M., Carmical, J. R., Starkey, J. M., et al. (2012). The national neuroAIDS tissue consortium brain gene array: two types of HIV-associated neurocognitive impairment. PLoS ONE 7:e46178. doi: 10.1371/journal.pone.0046178

Gelman, B. B., and Schuenke, K. (2004). Brain aging in acquired immunodeficiency syndrome: increased ubiquitin-protein conjugate is correlated with decreased synaptic protein but not amyloid plaque accumulation. J. Neurovirol. 10, 98108. doi: $10.1080 / 13550280490279816$

Gonzalez-Scarano, F., and Martin-Garcia, J. (2005). The neuropathogenesis of AIDS. Nat. Rev. Immunol. 5, 69-81. doi: 10.1038/nri1527

Gurwell, J. A., Nath, A., Sun, Q., Zhang, J., Martin, K. M., Chen, Y., et al. (2001). Synergistic neurotoxicity of opioids and human immunodeficiency virus-1 Tat protein in striatal neurons in vitro. Neuroscience 102, 555-563. doi: 10.1016/S0306-4522(00)00461-9

Hanada, T., Noda, N. N., Satomi, Y., Ichimura, Y., Fujioka, Y., Takao, T., et al. (2007). The Atg12-Atg5 conjugate has a novel E3-like activity for protein lipidation in autophagy. J. Biol. Chem. 282, 37298-37302. doi: 10.1074/jbc.C700195200

Hauser, K. F., El-Hage, N., Buch, S., Berger, J. R., Tyor, W. R., Nath, A., et al. (2005). Molecular targets of opiate drug abuse in neuroAIDS. Neurotox. Res. 8, 63-80. doi: 10.1007/BF03033820

Hauser, K. F., El-Hage, N., Stiene-Martin, A., Maragos, W. F., Nath, A. Persidsky, Y., et al. (2007). HIV-1 neuropathogenesis: glial mechanisms revealed through substance abuse. J. Neurochem. 100, 567-586. doi: 10.1111/j.14714159.2006.04227.x

Hauser, K. F., Fitting, S., Dever, S. M., Podhaizer, E. M., and Knapp, P. E. (2012). Opiate drug use and the pathophysiology of neuroAIDS. Curr. HIV Res. 10, 435-452. doi: 10.2174/157016212802138779

Irizarry, R. A., Hobbs, B., Collin, F., Beazer-Barclay, Y. D., Antonellis, K. J., Scherf, U., et al. (2003). Exploration, normalization, and summaries of high density oligonucleotide array probe level data. Biostatistics 4, 249-264. doi: 10.1093/biostatistics/4.2.249

Jaffe, J. H., and Martin, W. R. (1985). "Opioid analgesics and antagonists," in The Pharmacological Basis of Therapeutics, 7 Edn, eds A. G. Gilman, L. S. Goodman, T. W. Rall, and F. Murad (New York, NY: MacMillan Publishing Company), 491-531.

Kabeya, Y., Mizushima, N., Ueno, T., Yamamoto, A., Kirisako, T., Noda, T., et al. (2000). LC3, a mammalian homologue of yeast Apg8p, is localized in autophagosome membranes after processing. EMBO J. 19, 5720-5728. doi: 10.1093/emboj/19.21.5720

Kabeya, Y., Mizushima, N., Yamamoto, A., Oshitani-Okamoto, S., Ohsumi, Y., and Yoshimori, T. (2004). LC3, GABARAP and GATE16 localize to autophagosomal membrane depending on form-II formation. J. Cell Sci. 117, 2805-2812. doi: 10.1242/jcs.01131

Kaul, M., Garden, G. A., and Lipton, S. A. (2001). Pathways to neuronal injury and apoptosis in HIV-associated dementia. Nature 410, 988-994. doi: $10.1038 / 35073667$

Kaul, M., Zheng, J., Okamoto, S., Gendelman, H. E., and Lipton, S. A. (2005). HIV1 infection and AIDS: consequences for the central nervous system. Cell Death. Differ. 12(Suppl. 1), 878-892. doi: 10.1038/sj.cdd.4401623

Kimura, S., Noda, T., and Yoshimori, T. (2007). Dissection of the autophagosome maturation process by a novel reporter protein, tandem fluorescent-tagged LC3. Autophagy 3, 452-460. doi: 10.4161/auto.4451

Klionsky, D. J. (2012). Look people, "Atg" is an abbreviation for "autophagyrelated." That's it. Autophagy 8, 1281-1282. doi: 10.4161/auto.21812

Klionsky, D. J., Abeliovich, H., Agostinis, P., Agrawal, D. K., Aliev, G., Askew, D. S., et al. (2008). Guidelines for the use and interpretation of assays for monitoring autophagy in higher eukaryotes. Autophagy 4, 151-175. doi: 10.4161/auto.5338

Klionsky, D. J., and Emr, S. D. (2000). Autophagy as a regulated pathway of cellular degradation. Science 290, 1717-1721. doi: 10.1126/science.290.5497.1717

Komatsu, M., Ueno, T., Waguri, S., Uchiyama, Y., Kominami, E., and Tanaka, K. (2007a). Constitutive autophagy: vital role in clearance of unfavorable proteins in neurons. Cell Death. Differ. 14, 887-894. doi: 10.1038/sj.cdd.4402120

Komatsu, M., Waguri, S., Koike, M., Sou, Y. S., Ueno, T., Hara, T., et al. (2007b). Homeostatic levels of p62 control cytoplasmic inclusion body formation in autophagy-deficient mice. Cell 131, 1149-1163. doi: 10.1016/j.cell.2007.10.035

Kramer-Hammerle, S., Rothenaigner, I., Wolff, H., Bell, J. E., and BrackWerner, R. (2005). Cells of the central nervous system as targets and reservoirs of the human immunodeficiency virus. Virus Res. 111, 194-213. doi: 10.1016/j.virusres.2005.04.009 
Leshner, A. I. (1998). HIV prevention with drug using populations. Current status and future prospects. Public Health Rep. 113(Suppl. 1), 1-3.

Liang, X. H., Jackson, S., Seaman, M., Brown, K., Kempkes, B., Hibshoosh, H., et al. (1999). Induction of autophagy and inhibition of tumorigenesis by beclin 1 . Nature 402, 672-676. doi: 10.1038/45257

Livak, K. J., and Schmittgen, T. D. (2001). Analysis of relative gene expression data using real-time quantitative PCR and the 2(-Delta Delta C(T)) Method. Methods 25, 402-408. doi: 10.1006/meth.2001.1262

Mizushima, N., Yoshimori, T., and Levine, B. (2010). Methods in mammalian autophagy research. Cell 140, 313-326. doi: 10.1016/j.cell.2010.01.028

Moreau, K., Luo, S., and Rubinsztein, D. C. (2010). Cytoprotective roles for autophagy. Curr. Opin. Cell Biol. 22, 206-211. doi: 10.1016/j.ceb.2009. 12.002

Morgello, S., Gelman, B. B., Kozlowski, P. B., Vinters, H. V., Masliah, E., Cornford, M., et al. (2001). The national neuroAIDS tissue consortium: a new paradigm in brain banking with an emphasis on infectious disease. Neuropathol. Appl. Neurobiol. 27, 326-335. doi: 10.1046/j.03051846.2001. 00334.x

Nabha, L., Duong, L., and Timpone, J. (2013). HIV-associated neurocognitive disorders: perspective on management strategies. Drugs 73, 893-905. doi: 10.1007/s40265-013-0059-6

Nakaso, K., Yoshimoto, Y., Nakano, T., Takeshima, T., Fukuhara, Y., Yasui, K., et al. (2004). Transcriptional activation of p62/A170/ZIP during the formation of the aggregates: possible mechanisms and the role in Lewy body formation in Parkinson's disease. Brain Res. 1012, 42-51. doi: 10.1016/j.brainres.2004. 03.029

Nardacci, R., Amendola, A., Ciccosanti, F., Corazzari, M., Esposito, V., Vlassi, C., et al. (2014). Autophagy plays an important role in the containment of HIV-1 in nonprogressor-infected patients. Autophagy 10, 1167-1178. doi: 10.4161/auto. 28678

Nath, A., Conant, K., Chen, P., Scott, C., and Major, E. O. (1999). Transient exposure to HIV-1 Tat protein results in cytokine production in macrophages and astrocytes. A hit and run phenomenon. J. Biol. Chem. 274, 17098-17102. doi: $10.1074 / j b c .274 .24 .17098$

Pankiv, S., Clausen, T. H., Lamark, T., Brech, A., Bruun, J. A., Outzen, H., et al. (2007). p62/SQSTM1 binds directly to Atg8/LC3 to facilitate degradation of ubiquitinated protein aggregates by autophagy. J. Biol. Chem. 282, 2413124145. doi: $10.1074 /$ jbc.M702824200

Podhaizer, E. M., Zou, S., Fitting, S., Samano, K. L., El-Hage, N., Knapp, P. E., et al. (2012). Morphine and gp120 toxic interactions in striatal neurons are dependent on HIV-1 strain. J. Neuroimmune Pharmacol. 7, 877-891. doi: 10.1007/s11481-011-9326-z

Saeed, A. I., Bhagabati, N. K., Braisted, J. C., Liang, W., Sharov, V., Howe, E. A., et al. (2006). TM4 microarray software suite. Methods Enzymol. 411, 134-193. doi: 10.1016/S0076-6879(06)11009-5

Saeed, A. I., Sharov, V., White, J., Li, J., Liang, W., Bhagabati, N., et al. (2003). TM4: a free, open-source system for microarray data management and analysis. Biotechniques 34, 374-378.

Singh, I. N., Goody, R. J., Dean, C., Ahmad, N. M., Lutz, S. E., Knapp, P. E., et al. (2004). Apoptotic death of striatal neurons induced by human immunodeficiency virus-1 Tat and gp120: differential involvement of caspase-3 and endonuclease G. J. Neurovirol. 10, 141-151. doi: 10.1080/13550280490441103

Smith, D. L., Pozueta, J., Gong, B., Arancio, O., and Shelanski, M. (2009). Reversal of long-term dendritic spine alterations in Alzheimer disease models. Proc. Natl. Acad. Sci. U.S.A. 106, 16877-16882. doi: 10.1073/pnas.0908706106

Smyth, G. K. (2004). Linear models and empirical bayes methods for assessing differential expression in microarray experiments. Stat. Appl. Genet. Mol. Biol. 3, 1-25. doi: 10.2202/1544-6115.1027
Steele, A. D., Henderson, E. E., and Rogers, T. J. (2003). Mu-opioid modulation of HIV-1 coreceptor expression and HIV-1 replication. Virology 309, 99-107. doi: 10.1016/S0042-6822(03)00015-1

Tanida, I., Tanida-Miyake, E., Ueno, T., and Kominami, E. (2001). The human homolog of Saccharomyces cerevisiae Apg7p is a Protein-activating enzyme for multiple substrates including human Apg12p, GATE-16, GABARAP, and MAP-LC3. J. Biol. Chem. 276, 1701-1706. doi: 10.1074/jbc.C000752200

Tanida, I., Yamasaki, M., Komatsu, M., and Ueno, T. (2012). The FAP motif within human ATG7, an autophagy-related E1-like enzyme, is essential for the E2-substrate reaction of LC3 lipidation. Autophagy 8, 88-97. doi: 10.4161/auto.8.1.18339

Verma, A. S., Singh, U. P., Dwivedi, P. D., and Singh, A. (2010). Contribution of CNS cells in NeuroAIDS. J. Pharm. Bioallied Sci. 2, 300-306. doi: 10.4103/09757406.72129

Wang, Q. J., Ding, Y., Kohtz, D. S., Mizushima, N., Cristea, I. M., Rout, M. P., et al. (2006). Induction of autophagy in axonal dystrophy and degeneration. J. Neurosci. 26, 8057-8068. doi: 10.1523/JNEUROSCI.2261-06.2006

Wang, X., Gao, Y., Tan, J., Devadas, K., Ragupathy, V., Takeda, K., et al. (2012). HIV-1 and HIV-2 infections induce autophagy in Jurkat and CD4+ T cells. Cell. Signal. 24, 1414-1419. doi: 10.1016/j.cellsig.2012.02.016

Woods, S. P., Moore, D. J., Weber, E., and Grant, I. (2009). Cognitive neuropsychology of HIV-associated neurocognitive disorders. Neuropsychol. Rev. 19, 152-168. doi: 10.1007/s11065-009-9102-5

Wright, C. I. (1940). The enzymatic deacetylation of heroin and closely related morphine derivatives by blood serum. Science 92, 244-245. doi: 10.1126/science.92.2385.244-a

Yu, W. H., Cuervo, A. M., Kumar, A., Peterhoff, C. M., Schmidt, S. D., Lee, J. H., et al. (2005). Macroautophagy-a novel Beta-amyloid peptidegenerating pathway activated in Alzheimer's disease. J. Cell Biol. 171, 87-98. doi: $10.1083 /$ jcb. 200505082

Zatloukal, K., Stumptner, C., Fuchsbichler, A., Heid, H., Schnoelzer, M., Kenner, L., et al. (2002). p62 Is a common component of cytoplasmic inclusions in protein aggregation diseases. Am. J. Pathol. 160, 255-263. doi: 10.1016/S00029440(10)64369-6

Zhou, D., Masliah, E., and Spector, S. A. (2011). Autophagy is increased in postmortem brains of persons with HIV-1-associated encephalitis. J. Infect. Dis. 203, 1647-1657. doi: 10.1093/infdis/jir163

Zhou, D., and Spector, S. A. (2008). Human immunodeficiency virus type-1 infection inhibits autophagy. AIDS 22, 695-699. doi: 10.1097/QAD.0b013e $3282 \mathrm{f} 4 \mathrm{a} 836$

Zink, W. E., Zheng, J., Persidsky, Y., Poluektova, L., and Gendelman, H. E. (1999). The neuropathogenesis of HIV-1 infection. FEMS Immunol. Med. Microbiol. 26, 233-241. doi: 10.1111/j.1574-695X.1999.tb01394.x

Zou, S., Fitting, S., Hahn, Y. K., Welch, S. P., El-Hage, N., Hauser, K. F., et al. (2011). Morphine potentiates neurodegenerative effects of HIV-1 Tat through actions at mu-opioid receptor-expressing glia. Brain 134, 3616-3631. doi: 10.1093/brain/awr281

Conflict of Interest Statement: The authors declare that the research was conducted in the absence of any commercial or financial relationships that could be construed as a potential conflict of interest.

Copyright (๑) 2015 Dever, Rodriguez, Lapierre, Costin and El-Hage. This is an openaccess article distributed under the terms of the Creative Commons Attribution License (CC BY). The use, distribution or reproduction in other forums is permitted, provided the original author(s) or licensor are credited and that the original publication in this journal is cited, in accordance with accepted academic practice. No use, distribution or reproduction is permitted which does not comply with these terms. 